\title{
A COUNTEREXAMPLE TO QUESTIONS ABOUT BOUNDARIES, STABILITY, AND COMMENSURABILITY
}

\author{
JASON BEHRSTOCK
}

\begin{abstract}
We construct a family of right-angled Coxeter groups which provide counter-examples to questions about the stable boundary of a group, one-endedness of stable subgroups, and the commensurability types of right-angled Coxeter groups.
\end{abstract}

\section{INTRODUCTION}

In this short note we construct right-angled Coxeter groups with some interesting properties. These examples show that a number of questions in geometric group theory have more nuanced answers than originally expected. In particular, these examples resolve the following questions in the negative:

- (Charney and Sisto): As is the case for right-angled Artin groups, do all (nonrelatively hyperbolic) right-angled Coxeter group have totally disconnected contracting boundary?

- (Taylor): Given that all known quasigeodesically stable subgroups of the mapping class group are virtually free, does it hold that in any (non-relatively hyperbolic) group that all quasigeodesically stable subgroups have more than one end?

- (Folk question): If a right-angled Coxeter group has quadratic divergence, must it be virtually a right-angled Artin group?

We describe a family of graphs, any one of which is the presentation graph of a right-angled Coxeter group which provides a counterexample to all three of the above questions. We expect that in special cases, and perhaps in general with appropriate modifications, there are interesting positive answers to these questions; we hope this note will encourage the careful reader to formulate and prove such results.

The construction we give was inspired by thinking about the simplicial boundary for the Croke-Kleiner group, see [BH, Example 5.12] and [Tat2]. In the process we give a quick introduction to a few topics of recent interest in geometric group theory, for further details on these topics see also [ABD, BDM, Cha, CS, Cor2, DT, Tra].

Acknowledgments. Thanks to Ruth Charney, Alessandro Sisto, and Sam Taylor for sharing with me their interesting questions and to the referee for some helpful comments which led to Remark 2.3. Also, thanks to Mark Hagen for many fun discussions relating to topics in this note. 


\section{THE CONSTRUCTION}

Let $\Gamma_{n}$ be a graph with $2 n$ vertices built in the following inductive way. Start with a pair of vertices $a_{1}, b_{1}$ with no edge between them. Given the graph $\Gamma_{n-1}$, obtain the graph $\Gamma_{n}$ by adding a new pair of vertices $a_{n}, b_{n}$ to the graph $\Gamma_{n-1}$ and adding four new edges, one connecting each of $\left\{a_{n-1}, b_{n-1}\right\}$ to each of $\left\{a_{n}, b_{n}\right\}$. See Figure 1 .
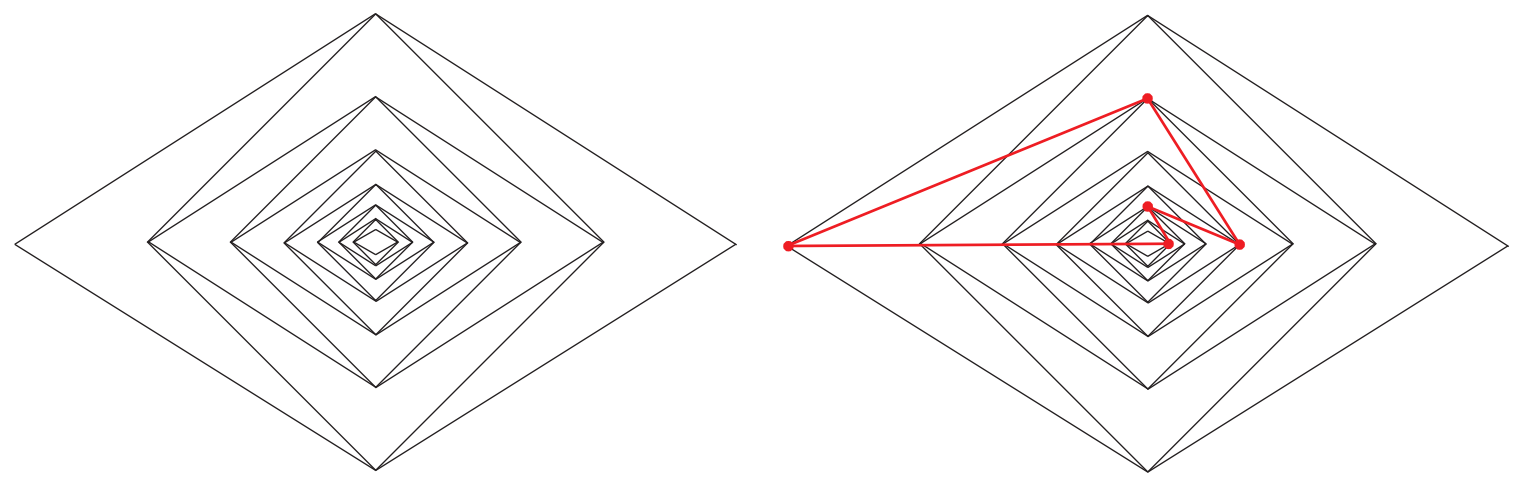

FiguRE 1. The graphs $\Gamma_{14}$ (left) and $\Gamma$ (right).

Note that $\Gamma_{n}$ is a join if and only if $n \leqslant 3$. More generally, $a_{i}, a_{j}$ are contained in a common join if and only if $|i-j| \leqslant 2$.

For any $m \geqslant 5$ choose $n$ sufficiently large so that there exists a set of points $\mathcal{P}=\left\{p_{1}, \ldots, p_{m}\right\} \subset \Gamma_{n}$ with the property that for each $1 \leqslant i<j \leqslant m$ the points $p_{i}$ and $p_{j}$ are not contained in a common join in $\Gamma_{n}$. For example, in $\Gamma_{14}$ we could choose the vertices $\mathcal{P}=\left\{a_{1}, a_{4}, a_{7}, a_{10}, a_{13}\right\}$. For each $1 \leqslant i<m$ add an edge between $p_{i}$ and $p_{i+1}$; also, add an edge between $p_{m}$ and $p_{1}$. Call this new graph $\Gamma$. There are many choices of $\Gamma$ depending on our choices of $n, m$, and $\mathcal{P}$; for the following any choice will work.

Associated to any graph, one can construct the right-angled Coxeter group with that presentation graph, this is the group whose defining presentation is given by: an order-two generator, for each vertex of the graph, and a commutation relation, between each of the generators associated to a pair of vertices connected by an edge.

Let $W$ denote the right-angled Coxeter group whose presentation graph is the $\Gamma$ constructed above. In the next section, we record some key properties about the group $W$ and then, in the final section, apply this to the questions in the introduction.

\section{PROperties}

\subsection{Quadratic divergence.}

Proposition 2.1. The group $W$ has quadratic divergence. In particular, this group is not relatively hyperbolic.

Proof. It is easily seen that the graph $\Gamma_{n}$ has the property that each vertex is contained in at least one induced square. It is also easy to verify that given any pair of induced squares $S, S^{\prime}$ in $\Gamma_{n}$, there exists a sequence of induced squares $S=S_{1}, S_{2}, S_{3}, \ldots, S_{k}=S^{\prime}$ where for each $1 \leqslant i<k$ the squares $S_{i}$ and $S_{i+1}$ share 3 vertices in common. This property, that there are enough squares to chain together 
any pair of points, is called $\mathcal{C} \mathcal{F S}$; it was introduced in [DaT] and studied further in [BFRHS, Lev].

Since $\Gamma$ has the same vertex set as $\Gamma_{n}$, and every induced square in $\Gamma_{n}$ is still induced in $\Gamma$, it follows that $\Gamma$ also has the $\mathcal{C F} \mathcal{S}$ property.

Given any graph with the $\mathcal{C F S}$ property and which is not a join, the associated right-angled Coxeter group has exactly quadratic divergence, see [DaT, Theorem 1.1] and [BFRHS, Proposition 3.1].

The second statement in the proposition follows from the fact that any relatively hyperbolic group has divergence which is at least exponential [Sis, Theorem 1.3].

2.2. Stable surface subgroups. An undistorted subgroup is said to be (quasi-geodesically) stable if each pair of points in that subgroup are connected by uniformly Morse quasigeodesics, see [DT].

Proposition 2.2. $W$ contains a closed hyperbolic surface subgroup which is stable.

Proof. Recall that in any right-angled Coxeter group an induced subgraph yields a subgroup isomorpic to the right-angled Coxeter group of the associated subgraph. Also, note that the right-angled Coxeter group associated to a cycle of length at least 5 is a 2-dimensional hyperbolic orbifold group. Thus, the subgraph spanned by $\mathcal{P}$, which is a cycle of length $m \geqslant 5$, yields a subgroup, $H$, which is isomorphic to a 2-dimensional hyperbolic orbifold group.

By construction, the subgraph $\mathcal{P}$ doesn't contain any pair of non-adjacent vertices in a common join of $\Gamma$ (by assumption we use the term join to mean non-degenerate join, in the sense that both parts of the join have diameter at least 2); thus no special subgroup of $H$ is a direct factor in a non-hyperbolic special subgroup of $W$.

It is proven in [ABD, Theorem B] that, a subgroup of a hierarchically hyperbolic space is stable if and only if it is undistorted and has uniformly bounded projections to the curve graph of every proper domain in some hierarchically hyperbolic structure; in turn, this property is equivalent to the orbit of the subgroup being quasi-isometrically embedded in the curve graph of the nest-maximal domain.

A hierarchically hyperbolic structure on right-angled Coxeter groups was built in [BHS]; in that structure the curve graphs are contact graphs which were first defined in [Hag1] where it was proven they are all quasi-trees. In the present context, a domain can be thought of as a certain convex subcomplex of the Davis complex arising from special subgroups and orthogonality holds when the Davis complex contains the direct product of a given such pair.

Since a closed surface group can not be quasi-isometrically embedded in a quasitree, to verify the proposition a different hierarchically hyperbolic structure must be used; for this we now rely on a method developed in [ABD] for modifying structures. The construction given in [ABD, Theorem 3.11] modifies the structure, by removing certain domains and augmenting the curve graphs associated to domains in which they are nested. Indeed, passing to the new structure and back, it follows that to verify a given subgroup has uniformly bounded projections in a particular structure, it suffices to verify that the subgroup has uniformly bounded projections to the set of domains $\mathcal{W}$ with the property that for each $W \in \mathcal{W}$, there exists a domain $U$ which is orthogonal to $W$ and with infinite diameter curve graph (since all other domains 
will be removed in the new structure while only changing the curve graph of the nest-maximal domain).

In the standard hierarchically hyperbolic structure the only domains to which $H$ has unbounded projections are contained in the subcomplex of the Davis complex associated to the special subgroup $H$. As noted above, since no pair of vertices in $\mathcal{P}$ are contained in a common join in $\Gamma$, these domains all have the property that any domains orthogonal to one of them must have uniformly bounded diameter. Thus, applying the results of [ABD] just discussed, it follows that $H$ is stable.

Now take a cover of the orbifold to get the desired closed hyperbolic surface.

Remark 2.3. We note that in the subgroup $H$ constructed in Proposition 2.2, it follows easily from [KL, Proposition 3.3] (or, more explicitly, from [Tra, Theorem 1.1]), that each infinite order element in $H$ acts as a rank-one isometry of the Davis complex of $W$ and is thus Morse. To see that such an argument alone is not enough to prove stability of the subgroup, we note that in [OOS] they construct lacunary hyperbolic groups which provide an obstruction. In particular, in [OOS, Theorem 1.12] it is proven that there exist infinite finitely generated non-hyperbolic groups in which every proper subgroup is infinite cyclic and generate uniformly Morse quasigeodesics.

The next result follows from Proposition 2.2 and [Cor1, Proposition 4.2].

Corollary 2.4. The right-angled Coxeter group $W$ contains a topologically embedded circle in its Morse boundary.

\section{ApplicATIONS}

3.1. Morse boundaries. Charney and Sultan introduced a boundary for CAT(0) groups which captures aspects of the negative curvature of the group [CS]. Their construction was then generalized by Cordes to a framework which exists for all finitely generated groups [Cor1]; in this general context it is known as the Morse boundary. Charney and Sultan built examples of relatively hyperbolic right-angled Coxeter groups whose boundaries are not totally disconnected [CS]. More generally, it is now known that for hyperbolic groups, the Morse boundary coincides with the hyperbolic boundary [Cor1, Main Theorem (3)]; using this it is easy to produce many examples of hyperbolic and relatively hyperbolic right-angled Coxeter groups whose boundary are not totally disconnected.

On the other hand, the Morse boundary of any right-angled Artin group $A_{\Gamma}$ is totally disconnected. The two-dimensional case of this is implicit in [CS]. In general, this fact follows from the fact that the contact graph has totally disconnected boundary (since it is a quasi-tree) and [CH, Theorem F]. To see this recall that $[\mathrm{CH}$, Theorem F] provides a continuous map from the Morse boundary of $A_{\Gamma}$ to the boundary of the contact graph of the universal cover of the Salvetti complex of $A_{\Gamma}$; at which point the result follows since Morse geodesic rays lying at infinite Hausdorff distance cannot fellow-travel in the contact graph, so this continuous boundary map is injective.

Accordingly, Ruth Charney and Alessandro Sisto raised the question of whether outside of the relatively hyperbolic setting, right-angled Coxeter groups all have totally disconnected Morse boundary. The group $W$ constructed above shows the 
answer is no, since it is not relatively hyperbolic by Proposition 2.1 and its boundary is not totally disconnected, by Corollary 2.4 .

3.2. Stable subgroups. Examples of stable subgroups are known both in the mapping class group [Beh, DT] and in right-angled Artin groups [KMT]. In both of these classes, all known examples of stable subgroups are virtually free; in the relatively hyperbolic setting on the other hand, it is easy to construct one-ended stable subgroups. Sam Taylor asked whether there exist non-relatively hyperbolic groups with one-ended stable subgroups. The example $W$ is a non-relatively hyperbolic group with one-ended stable subgroups by Proposition 2.1 and Proposition 2.2.

3.3. Commensurability. A well-known construction of Davis-Januszkiewicz [DJ] shows that every right-angled Artin group is commensurable to some right-angled Coxeter group. The following is a well-known problem:

Question 3.1. Which right-angled Coxeter groups are commensurable to rightangled Artin groups?

It is known that any right-angled Artin groups either has divergence which is linear (if it is a direct product) or quadratic, see $[\mathrm{BC}]$ or $\left[\mathrm{ABD}^{+}\right]$. Since divergence is invariant under quasi-isometry, and hence under commensurability as well, this puts a constraint on the answer to Question 3.1. Several people have raised the question of whether every right-angled Coxeter group with quadratic divergence is quasi-isometric to some right-angled Artin group. The group $W$ shows that the answer is no, since [Cor1, Main Theorem (2)] proves that the Morse boundary is invariant under quasi-isometries, but Propositions 2.1 and 2.2 show that the group $W$ is a right-angled Coxeter group with quadratic divergence whose Morse boundary contains an embedded circle, while the Morse boundary of any right-angled Artin group is totally disconnected.

\section{FURTHER QUESTIONS}

In [BFRHS] it was established that for a large range of density functions that asymptotically almost surely the random graph yields a right-angled Coxeter group with quadratic divergence. More generally, it is known that among $n$-vertex graphs with density greater than $1 / n$ and bounded away from 1 , that asymptotically almost surely any random graph of this type will contain a large induced polygon. Accordingly, we expect that the proof of Corollary 2.4 could be used to verify:

Conjecture 4.1. For any density greater than $1 / n$ and bounded away from 1 , asymptotically almost surely the random right-angled Coxeter group contains circles in its Morse boundary. In particular, it is not virtually a right-angled Artin group.

The prevalence of right-angled Coxeter groups with quadratic divergence makes the following an appealing (but likely very difficult) question:

Question 4.2. Classify right-angled Coxeter groups with quadratic divergence up to quasi-isometry. Classify them up to commensurability.

The proof of Proposition 2.2 and Remark 2.3 indicate that there are some nontrivial constraints on what the set of domains could be in any potential hierarchically hyperbolic structure on a lacunary hyperbolic group. Accordingly we ask: 
Question 4.3. Is a lacunary hyperbolic group hierarchically hyperbolic if and only if it is hyperbolic?

Further, in light of Proposition 2.2 and Remark 2.3 it would be interesting if the following was true in general or under some moderate hypotheses:

Question 4.4. Let $G$ be a hierarchically hyperbolic group with a subgroup $H$. If all infinite cyclic subgroups of $H$ are uniformly Morse, does that imply that $H$ is stable?

\section{REFERENCES}

[ABD] Carolyn Abbott, Jason Behrstock, and Matthew G Durham. Largest acylindrical actions and stability in hierarchically hyperbolic groups. ARXIV:1705.06219.

$\left[\mathrm{ABD}^{+}\right]$A. Abrams, N. Brady, P. Dani, M. Duchin, and R. Young. Pushing fillings in right-angled Artin groups. J. Lond. Math. Soc. (2) 87 (2013), 663-688.

[Beh] J. Behrstock. Asymptotic geometry of the mapping class group and Teichmüller space. Geometry E Topology 10 (2006), 1523-1578.

[BC] J. Behrstock and R. Charney. Divergence and quasimorphisms of right-angled Artin groups. Math. Ann. 352 (2012), 339-356.

[BDM] J. Behrstock, C. Druţu, and L. Mosher. Thick metric spaces, relative hyperbolicity, and quasi-isometric rigidity. Math. Ann. 344 (2009), 543-595.

[BFRHS] J. Behrstock, V. Falgas-Ravry, M. Hagen, and T. Susse. Global structural properties of random graphs. Int. Math. Res. Not. (2016). To appear.

$[\mathrm{BH}] \quad$ J. Behrstock and M.F. Hagen. Cubulated groups: thickness, relative hyperbolicity, and simplicial boundaries. Geometry, Groups, and Dynamics 10 (2016), 649-707.

[BHS] J. Behrstock, M.F. Hagen, and A. Sisto. Hierarchically hyperbolic spaces I: curve complexes for cubical groups. Geometry \& Topology, 21:1731-1804, 2017.

[Cha] Ruth Charney. An introduction to right-angled Artin groups. Geom. Dedicata 125 (2007), 141-158.

[CS] Ruth Charney and Harold Sultan. Contracting boundaries of CAT(0) spaces. J. Topol. 8 (2015), 93-117.

[Cor1] Matthew Cordes. Morse boundaries of proper geodesic metric spaces. ARXIV:1502.04376.

[Cor2] Matthew Cordes. A survey on Morse boundaries \& stability. ARXIV:1704.07598.

$[\mathrm{CH}]$ Matthew Cordes and David Hume. Stability and the Morse boundary. To appear in Groups, Geometry, and Dynamics (2016).

[DaT] Pallavi Dani and Anne Thomas. Divergence in right-angled Coxeter groups. Trans. Amer. Math. Soc. 367 (2015), 3549-3577.

[DJ] Michael W. Davis and Tadeusz Januszkiewicz. Right-angled Artin groups are commensurable with right-angled Coxeter groups. J. Pure Appl. Algebra 153 (2000), 229-235.

[DT] Matthew Gentry Durham and Samuel J. Taylor. Convex cocompactness and stability in mapping class groups. Algebr. Geom. Topol. 15 (2015), 2839-2859.

[Hag1] Mark F. Hagen. Weak hyperbolicity of cube complexes and quasi-arboreal groups. J. Topol. 7 (2014), 385-418.

[KL] M. Kapovich and B. Leeb. 3-manifold groups and nonpositive curvature. Geom. Funct. Anal. 8 (1998), 841-852.

[KMT] Thomas Koberda, Johanna Mangahas, and Samuel J Taylor. The geometry of purely loxodromic subgroups of right-angled Artin groups. ARXIV:1412.3663.

[Lev] Ivan Levcovitz. Divergence of CAT(0) Cube Complexes and Coxeter Groups. arXiv:1611.04378.

[OOS] Alexander Yu. Ol'shanskii, Denis V. Osin, and Mark V. Sapir. Lacunary hyperbolic groups. Geom. Topol. 13(2009), 2051-2140. With an appendix by Michael Kapovich and Bruce Kleiner.

[Sis] A. Sisto. On metric relative hyperbolicity. ARXIV:1210.8081.

[Tat2] Mark F. Hagen. Tattoo. In preparation.

[Tra] Hung Cong Tran. Purely loxodromic subgroups in right-angled Coxeter groups. ARXIV:1703.09032. 
Lehman College and The Graduate Center, CUNY, New York, New York, USA

E-mail address: jason.behrstock@lehman. cuny.edu 